\title{
Contributions of the 12 Segments of Left Ventricular Myocardium to the Body Surface Potentials
}

\author{
Juho Väisänen ${ }^{1}$, Jesús Requena-Carrión ${ }^{2}$, Felipe Alonso-Atienza ${ }^{2}$, Jari Hyttinen ${ }^{1}$, \\ José Luis Rojo-Álvarez ${ }^{2}$, and Jaakko Malmivuo ${ }^{1}$ \\ ${ }^{1}$ Ragnar Granit Institute, Tampere University of Technology, Tampere, Finland \\ juho.vaisanen@tut.fi \\ ${ }^{2}$ Teoría de la Señal y Comunicaciones, Universidad Carlos III, \\ Leganés, Spain
}

\begin{abstract}
Experimental and inverse approaches have been applied in studying the contributions of different parts of the myocardium to the ECG measurements. Also optimal electrode locations for different clinical purposes have been studied by applying body surface maps. It is valuable to know where the measured ECG is actually generated. Thus the measurements can be designed to be most optimal to measure certain myocardial sources. Here we assess the contributions of 12 left ventricular segments to the potentials of 117 surface leads. The study is based on the numerical lead field analysis combined with the cardiac activation modeling. We analyzed the contributions of the signals generated by different segments to the total signal generated by the left ventricle. It was found that anterior segments have high contributions to the leads on the lower left thorax and inferior segments on the leads on the lower left back. These results were expected based on the previous clinical studies.
\end{abstract}

\section{Introduction}

Today various electrode systems are applied in measuring the electrical activity of myocardium. The properties and benefits of these electrocardiography (ECG) lead systems, such as multielectrode systems with 24 to 190 electrodes or standard 12-lead ECG have been studied widely. All the studies have tried to answer, one way or another the questions; How many leads are needed for different measurement purposes and where they should be located. Trägårdh and colleagues [1] have recently published a good review of these clinical studies. In the present paper we study the contributions of 12 left ventricular segments to the body surface potentials measured in 117 leads, i.e. which source locations within the left ventricular myocardium are actually measured by each lead. The analysis is based on the sensitivity distributions of surface electrodes and simulated cardiac activation.

The past body surface potential map (BSPMs) studies have suggested optimal electrode locations for different clinical cases such as detection of infarctions and ischemia [2-4]. Many of these studies suggest electrode locations which are specific to measure and indicate changes in the activation of different parts of myocardium such as anterior segments of left ventricle. The studies of optimal measurement leads 
to detect different cardiac abnormalities have been mainly conducted by statistical data analysis of measured BSMPs [5-7]. The statistical methods and BSPMs are very applicable when surface measurements are studied and evaluated. Despite of their efficiency these methods might be impractical or even unuseful when designing and optimizing new measurements and devices such as implantable ECG monitors.

The true origins of the electrophysiological phenomena measured are of interest in many cases. Inverse problems and experimental setups have been applied in studying the effects of spatial and temporal characteristics of cardiac activation on the body surface potentials [8-12]. For example MacLeod and colleagues found in [8] that some locations of infarcts are more visible in some body surface potentials than in others and there exist so called silent changes in cardiac activation which are not projected to the body surface. These studies suggest that there are electrode locations which are sensitive to activation arisen in certain areas of myocardium.

When designing novel measurement devices and setups it would be beneficial to know where to locate the electrodes to measure the target signals and monitor their source regions as efficiently as possible. One might also desire to concentrate the measurement in a certain area of the cardiac muscle, like in a segment of the left ventricle. Thus the electrodes should be located in such places that the measurement is most specific to the activation arising in this region of interest. This is especially of interest when monitoring different cardiac arrhythmias or changes in activation of certain segment of myocardium after infarctions. Also designing of active cardiac devices such as implantable defibrillators and pacemakers could benefit of such approach where the measurement and stimulating lead configurations could be studied by modeling approach.

Modeling of measurements and their sensitivity distributions together with simulation of cardiac activity serves time and cost effective approach to study the properties of measurements. In the past only few methods have been applied in analyzing the sensitivity distributions of measurement setups. Thus the possibilities of sensitivity distribution analysis have not been utilized as efficiently as possible. The lead field and reciprocity approaches provide effective tools to analyze the sensitivity distributions. In this paper we apply the lately developed method which combines the lead field approach with dynamic source model of cardiac activation in studying the origins of the body surface potentials. The sensitivity distributions were calculated in a realistic model of human thorax by applying finite difference method (FDM). We simulated the dynamic source distribution in left ventricular myocardium and calculated the lead fields of 117 body surface leads. Signals in these leads can be solved by combining the lead fields and source distributions. In [13] we have presented a method to analyze the contribution of certain source volume to the measured signal by estimating the mean square difference (MSD) between signal generated by these sources and the signal generated by the whole source volume. Here we studied the contributions of 12 segments of left ventricle to the source volume which generates signal having $10 \%$ MSD when compared to the signal generated by whole source volume. This analysis was applied to all leads and thus we can estimate which electrode location is most optimal to measure different segments of left ventricular myocardium with the applied activation model. 


\section{Material and Methods}

\subsection{Finite Difference Method}

In the FDM the segmented volume data, e.g. from an MRI dataset, are divided into cubic elements forming a resistive network. In short the resistances of the elements correspond to the tissue resistivities and the dimensions of the elements correspond to the resolution of the dataset. The network can be described as a set of linear equations which present the potentials and currents in the model. The FDM is based on the Poisson's equation that can be used to describe the bioelectric quasistatic source-field problems [14]. FDM models are discrete and thus continuous Poisson's functions have to be discretized to form linear equations. A potential distribution within the model for a specific source configuration is solved with these linear equations with iterative methods [15]. The use of FDM method in modeling sensitivity distributions is validated in [16].

\section{Realistic model of human anatomy}

The FDM allows the implementation of complex anatomic geometries from the image data, and the resulting potentials and currents can be calculated within the whole volume conductor model [15]. In the present study we applied a FDM model of the 3D male thorax based on the Visible Human Man dataset (VHM) [17, 18]. The applied dataset represents data on 95 segmented slices where resolution in the slices close to the heart was $1.67 \mathrm{~mm} \times 1.67 \mathrm{~mm} \times 4 \mathrm{~mm}$ and elsewhere $1.67 \mathrm{~mm} \times 1.67 \mathrm{~mm}$ x $8 \mathrm{~mm}$. Model contains altogether 2.7 million nodes with 2.6 million elements. The model applied contains over 20 different organ and tissue types with corresponding resistivities which are listed in Table 1 [16]. Figure 1 illustrates the model with 117 electrode locations applied in the study.

Table 1. Resistivity values in the model. Blood masses include atrium, ventricles, aortic arch, ascending aorta, descending aorta, superior vena cava, inferior vena cava, carotid artery, jugular vein, pulmonary artery, pulmonary vein and other blood.

\begin{tabular}{cccc}
\hline \hline Organ/tissue & $\begin{array}{c}\text { Resistivity } \\
(\mathbf{\Omega} \mathbf{c m})\end{array}$ & Organ/tissue & $\begin{array}{c}\text { Resistivity } \\
(\mathbf{\Omega} \mathbf{c m})\end{array}$ \\
\hline Air & $10^{10}$ & Kidney & 600 \\
Skeletal muscle & 400 & Liver & 600 \\
Fat & 2000 & Heart muscle & 450 \\
Bone & 2000 & Heart fat & 2000 \\
Lungs & 1325 & Blood masses* & 150 \\
Stomach & 400 & $\begin{array}{l}\text { Other tissues and } \\
\text { organs on thorax }\end{array}$ & 460 \\
\hline \hline
\end{tabular}

\subsection{Lead Field and Reciprocity}

The sensitivity distribution of the measurement configurations can be illustrated and analyzed with lead fields. Lead fields are thus applied to describe and study the 

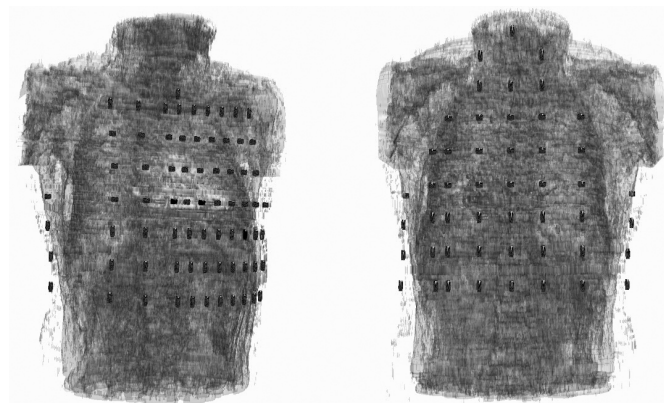

Fig. 1. 3D model of torso containing 117 electrode locations

measurement of the electric fields in the volume conductor. Lead current density vectors define the relationship between the measured potential in the lead and the current sources in the volume conductor following Equation 1. The measured lead voltage is dependent on the magnitudes of the lead and source current vectors as well as the angle between these vectors [14].

$$
z[n]=\sum_{V} \frac{1}{\sigma} \frac{1}{I_{r}} \bar{J}_{L} \cdot \bar{J}^{i}[n]
$$

Where $z[n]$ is the lead voltage as a function of time $n, \bar{J}_{L}$ is the lead current density vector $\left[\mathrm{A} / \mathrm{cm}^{2}\right], I_{r}$ is the applied reciprocal current $[\mathrm{A}], \bar{J}^{i}[n]$ is the current source density vector $\left[\mathrm{A} / \mathrm{cm}^{2}\right]$ as a function of time, $\sigma$ is the conductivity $[1 / \Omega \mathrm{cm}]$ of the source location in the volume conductor and $V$ is the source volume.

The lead field in the volume conductor can be established by applying the principle of reciprocity. In [14] it is stated that the current field in the volume conductor raised by the reciprocal unit current $\left(\mathrm{I}_{\mathrm{r}}=1\right.$ A) applied to the measurement electrodes corresponds to the lead current density and hence to the lead field. The essential benefit of this method is that the sensitivity of a measurement lead at all source locations in the volume conductor can be calculated with a single calculation.

\subsection{Cardiac Activation Model}

In our implementation we opted for a state machine approach defined previously in [19]. This model of cardiac electric activity reproduces electric restitution of both action potential duration (APD) and conduction velocity (CV), as well as curvature effects. Cardiac tissue is modeled as a grid of discrete elements characterized by three discrete states, namely, Rest, Refractory 1 and Refractory 2 , and transitions among them. The excitation of an element, i.e. the transition from Rest to Refractory 1 is interpreted as a probabilistic event, depending on the amount of excitation in its neighborhood, and the excitability of the element, that can be accessed through the restitution curve of CV. Transitions from Refractory 1 to Rest through Refractory 2 depend on the current of APD. Additionally, a membrane voltage is assigned at every 
time instant. Finally, non-conservative sources at each time $n$ and location $i, \bar{J}^{i}[n]$, are solved based on the voltage differences and conductivities between neighboring elements.

\subsection{Mean Square Difference Estimating Contribution of the Sources to the Signal}

In [13] we introduced a method to analyze the contributions of the sources to the measured signal. The contribution of certain sources to the measured signal is defined by the mean square difference (MSD) between the signal generated by these sources and signal generated by whole source volume. Let $V$ be the total source volume and $V_{o}$ be subset of the sources within the $V$. Based on (1), we define that the ECG signal $z[n]$ is generated by $V$ and $z_{o}[n]$ is generated by the source in $V_{o}$. If we measure the similarity between $z[n]$ and $z_{0}[n]$ based on MSD, we obtain:

$$
M S D\left\{V_{o}\right\}=E\left[\left(z[n]-z_{o}[n]\right)^{2}\right]
$$

Furthermore, by normalizing MSD by the mean power of whole source volume $z[n], P_{z z}$, we can obtain a more convenient measurement of similarity relative to the mean power of $z[n]$ :

$$
\operatorname{MSD}_{n}\left\{V_{o}\right\}=\frac{\operatorname{MSD}\left\{V_{o}\right\}}{P_{z z}}
$$

\subsection{Calculations}

We calculated the sensitivity distributions, the lead fields, in a realistic model of the male thorax by applying the principle of reciprocity. The lead fields were calculated for standard 117 body surface electrode leads illustrated in Figure 2. The same lead arrangement has been adopted for example by Kornreich and colleagues [4, 20]. The current distribution corresponding to the lead field current density was generated into the volume conductor by applying the unit currents to the electrode pairs. The calculations were executed with bioelectric field software which applies the Incomplete Cholesky Preconditioner and Conjugate Gradient for solution [21].

Here we study the contributions of the sources of left ventricular segments to the signals measured with 117 leads. The left ventricle was divided into 12 segments based on the standard 12 segment left ventricular subdivision recommended by the Committee on Nomenclature of Myocardial Wall Segments of the International Society of Computerized Electrocardiography [22, 23] illustrated in Figure 3.

We solved source distribution $\bar{J}^{i}[n]$ for the activation starting on the apex and conducting through the left ventricle over one second. The source distribution was combined with the sensitivity distributions to solve the measured potentials in the surface leads as described by (1). For each lead we evaluated the source volume $V_{o}$ within the left ventricle which generates signal with $10 \% \mathrm{MSD}_{\mathrm{n}}$ compared to the signal generated by whole left ventricle. Further we observed the proportions of the 12 segments in $V_{o}$ and studied which segments have the largest influence on the signals measured with surface leads. 


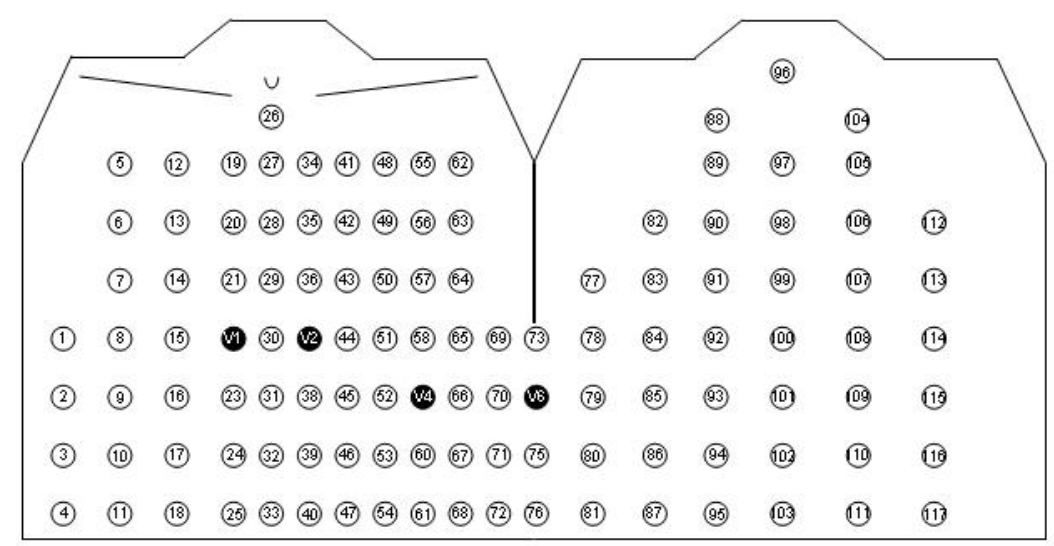

Fig. 2. 117 body surface electrode leads

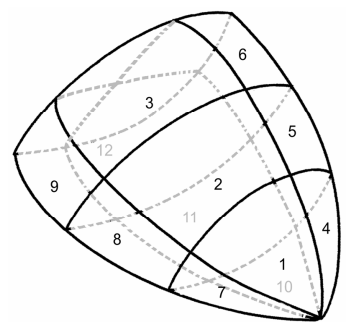

Fig. 3. The standard 12-segment left ventricular subdivision. Segments 1-3 anteroseptal, 4-6 anterosuperior, 7-9 inferior and 10-12 posterolateral.

\section{Results}

Figure 4 presents for 117 leads the proportions of the 12 left ventricular segments to the source volume which produces signal having $10 \% \mathrm{MSD}_{\mathrm{n}}$ when compared to the total signal generated by the left ventricle.

From the results we may observe the segments of the left ventricle which have the highest proportions of the sources measured with surface leads. Thus these leads are most optimal to measure activation generated within the segments. As an example over 30 percent of the sources that produce the signal with $10 \% \mathrm{MSD}_{\mathrm{n}}$ in the lead 93 are within the segment 8 . Further Segments 8 and 9 have together over $60 \%$ of the sources which produce the signal having $10 \% \mathrm{MSD}_{\mathrm{n}}$ in the lead 93.

There are leads that are most optimal to measure different segments. For example approximately $50 \%$ of the sources producing signal with $10 \% \mathrm{MSD}_{\mathrm{n}}$ in leads on the mid left thorax are in segments 1, 4 and 5 and fewer than $10 \%$ of the sources are within the segments 8,9 and 12 . Thus these leads are most optimal to measure the signal originating from anterior segments. Leads in lower left back are mainly 


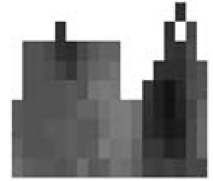

1

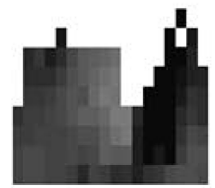

4

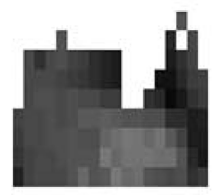

7

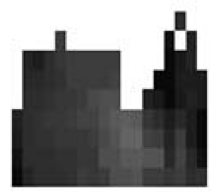

10

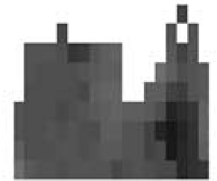

2

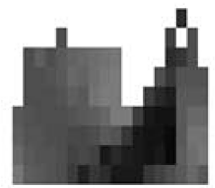

5

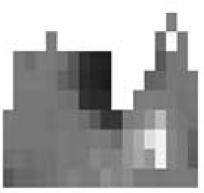

8

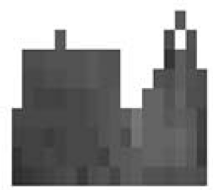

11

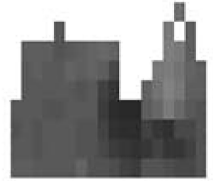

3

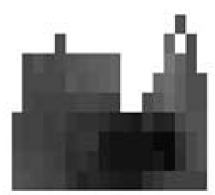

6

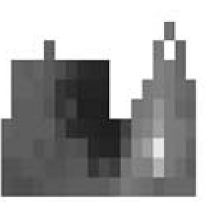

9

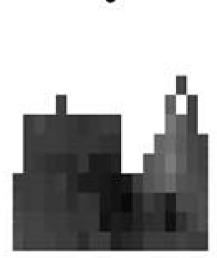

12

Fig. 4. For 117 leads the proportions of the 12 left ventricular segments in the volume generating signal with $10 \% \mathrm{MSD}_{\mathrm{n}}$ compare to the signal generated by whole left ventricle. The larger the proportion the higher is the contribution of the segment into the signal measured on the lead and more likely changes in measured signal are originating from the corresponding segment.

measuring the activation of segments 8 and 9 because these segments have approximately $60 \%$ share of the sources measured with these leads.

\section{Discussion}

Realistic and highly accurate models of the human anatomy are needed to carry out efficient modeling and simulation of the measurements leads. The FDM enables effective use of the models and thus leads to a more accurate modeling of measurements. Nevertheless the accuracy of the results can be affected by the resolution of the model and the number of conductivities modeled. These factors can affect the potential distribution within volume conductor and further the current density vectors which correspond to the sensitivity vectors. The higher the resolution of FDM grid the lower is the numerical error produced by discretization of 
conductivities and thus more accurate current density vectors are achieved. Numerical approach applied here takes into account double sided differences of potentials in calculation of sensitivities and thus decreases the numerical error produced by the discretization of conductivities.

The resolution of the FDM model defines the time needed for the calculation. A calculation of sensitivity distribution of a lead in this study took approximately 15 minutes with AMD 3000+64Bit, 2 GB RAM. Solving the one second of cardiac activation in left ventricle took less than 3 seconds with Intel P4 2,4GHz and $1.5 \mathrm{~GB}$ RAM. Models with higher resolution can be applied in studies with high computational resources without excessive computation times.

Although the model utilized possesses high anatomical accuracy, it has certain other shortcomings. In the present study we applied the finite difference model with a network of resistances featuring the tissue impedance. The human tissues also have capacitive characteristics possibly affecting the current distribution on the thorax. However, it has been recognized that the ratios between conductivities and permittivities of the human tissues on the frequency range of ECG are such that the dielectric behavior of tissues is mainly resistive [24]. Thus the model containing only resistive properties is relevant for our purposes.

Furthermore, the model applied in the present study is isotropic. Anisotropic conductivities, especially of the cardiac muscle would improve the simulations. The model was based on the anatomy of a single human subject and the segmentation of the tissues might also have some shortcomings. In the future we will apply other thorax models to study the effect of model on the results. Also the resolution and segmentation of models will be enhanced.

The MSD was found to be an efficient approach when the contributions of different segments were observed. In the present study we decided to observe the $10 \%$ difference but in future studies also other difference should be considered when the contributions of sources are observed. It would be valuable to investigate if the proportions are the same with the larger differences. Also other statistical properties of signals such as standard deviation and correlation will be included in the analysis in future studies.

Kornreich and colleagues have studied which are the most optimal leads to detect anterior and inferior myocardial infarctions. The anterior infarction volume contains segments 1-7, 10 and 11 which are supplied by left descending artery or its branches [23]. The inferior infarction volume contains segments 8 and 9 supplied by inferior descending artery or its proximate coronary [23]. Kornreich and colleagues found in [4] that the optimal leads to detect anterior infarction are located in the left thorax around leads 50 and 69. They also found that the inferior infarction is most optimally detected from the leads on the left lower back. The same conclusions can be obtained from our results because segments involved in anterior infarction have high contributions to the signals measured with leads on the mid-left thoracic region and segments involved in the inferior infarction have high contributions to the leads on lower left back.

Although the model and segmentation of left ventricle to 12-segments are quite coarse the study shows that there are locations which are more specific to measure individual segment and locations which are sensitive for number of segments. It is also shown that the modeling and the analyzing methods presented here provide excellent tools when studying the contribution of myocardial sources to the measured 
body surface potentials. In the present study only one type of activation model was applied. The activation model has impact on the results and in the future we will thus apply multiple activation models to study e.g. the effect of starting point of ectopic beat on the sensitivities of the leads. In the future this method will be also applied in studying and designing of implantable ECG monitors as well as analyzing the origins of signals measured with implantable cardiac defibrillators and pacemakers.

Acknowledgements. Authors would like to thank $\mathrm{PhD}$ Noriyuki Takano for providing the FDM solver software and MSc Tuukka Arola for providing FDM visualization tool. The work has been supported by grants from the Finnish Cultural Foundation Pirkanmaa Region and the Ragnar Granit Foundation.

\section{References}

1. Tragardh, E., Engblom, H., Pahlm, O.: How many ECG leads do we need? Cardiology Clinics 24 (3), 317-330 (2006)

2. Horacek, B.M., Warren, J.W., Penney, C.J., MacLeod, R.S., Title, L.M., Gardner, M.J., Feldman, C.L.: Optimal electrocardiographic leads for detecting acute myocardial ischemia. J. Electrocardiol 34 Suppl, 97-111 (2001)

3. Kornreich, F.: Identification of best electrocardiographic leads for diagnosing acute myocardial ischemia. J. Electrocardiol 31 Suppl, 157-163 (1998)

4. Kornreich, F., Montague, T.J., Rautaharju, P.M., Block, P., Warren, J.W., Horacek, M.B.: Identification of best electrocardiographic leads for diagnosing anterior and inferior myocardial infarction by statistical analysis of body surface potential maps. Am. J. Cardiol. 58(10), 863-871 (1986)

5. Kornreich, F., Montague, T.J., Rautaharju, P.M.: Body surface potential mapping of ST segment changes in acute myocardial infarction. Implications for ECG enrollment criteria for thrombolytic therapy. Circulation 87(3), 773-782 (1993)

6. Finlay, D.D., Nugent, C.D., Donnelly, M.P., Lux, R.L., McCullagh, P.J., Black, N.D.: Selection of optimal recording sites for limited lead body surface potential mapping: a sequential selection based approach. BMC Med. Inform. Decis. Mak. 6, 9 (2006)

7. Aldrich, H.R., Hindman, N.B., Hinohara, T., Jones, M.G., Boswick, J., Lee, K.L., Bride, W., Califf, R.M., Wagner, G.S.: Identification of the optimal electrocardiographic leads for detecting acute epicardial injury in acute myocardial infarction. Am. J. Cardiol. 59(1), 20-23 (1987)

8. MacLeod, R.S., Lux, R.L., Taccardi, B.: A possible mechanism for electrocardiographically silent changes in cardiac repolarization. J. Electrocardiol. 30 Suppl, 114-121 (1998)

9. Burnes, J.E., Taccardi, B., MacLeod, R.S., Rudy, Y.: Noninvasive ECG imaging of electrophysiologically abnormal substrates in infarcted hearts: A model study. Circulation 101(5), 533-540 (2000)

10. He, B., Li, G., Zhang, X.: Noninvasive imaging of cardiac transmembrane potentials within three-dimensional myocardium by means of a realistic geometry anisotropic heart model. IEEE Trans Biomed Eng. 50(10), 1190-1202 (2003)

11. Fukuoka, Y., Oostendorp, T.F., Sherman, D.A., Armoundas, A.A.: Applicability of the single equivalent moving dipole model in an infinite homogeneous medium to identify cardiac electrical sources: a computer simulation study in a realistic anatomic geometry torso model. IEEE Trans Biomed Eng. 53(12 Pt 1), 2436-2444 (2006) 
12. Brooks, D.H., MacLeod, R.S.: Electrical imaging of the heart. Signal Processing Magazine, IEEE 14(1), 24-42 (1997)

13. Requena Carrión, J., Väisänen, J., Rojo Álvarez, J.L., Hyttinen, J., Atienza, F.A., Malmivuo, J.: Numerical Analysis of the Resolution of Surface Electrocardiographic Lead Systems. In: proc. 4th Functional Imaging and Modeling of the Heart (2007)

14. Malmivuo, J., Plonsey, R.: Bioelectromagnetism: Principles and Applications of Bioelectric and Biomagnetic Fields. Oxford University Press, New York (1995)

15. Johnson, C.R.: Computational and numerical methods for bioelectric field problems. Crit Rev. Biomed. Eng. 25(1), 1-81 (1997)

16. Kauppinen, P., Hyttinen, J., Laarne, P., Malmivuo, J.: A software implementation for detailed volume conductor modelling in electrophysiology using finite difference method. Comput. Methods Programs Biomed. 58(2), 191-203 (1999)

17. Ackerman, M.J.: The Visible Human Project. J. Biocommun 18(2), 14 (1991)

18. Kauppinen, P., Hyttinen, J., Heinonen, T., Malmivuo, J.: Detailed model of the thorax as a volume conductor based on the visible human man data. J. Med. Eng. Technol. 22(3), 126-133 (1998)

19. Atienza, F.A., Carrion, J.R., Alberola, A.G., Alvarez, J.L.R., Munoz, J.J.S., Sanchez, J.M., Chavarri, M.V.: A probabilistic model of cardiac electrical activity based on a cellular automata system. Revista Espanola De. Cardiologia 58(1), 41-47 (2005)

20. Kornreich, F., Rautaharju, P.M., Warren, J., Montague, T.J., Horacek, B.M.: Identification of best electrocardiographic leads for diagnosing myocardial infarction by statistical analysis of body surface potential maps. Am. J. Cardiol. 56(13), 852-856 (1985)

21. Takano, N.: Reduction of ECG Leads and Equivalent Sources Using Orthogonalization and Clustering Techniques. in Ragnar Granit Institute Tampere University of Technology (2002)

22. Wagner, G.S., Cowan, M.J., Flowers, N.C., Ginzton, L.E., Ideker, R.E., Laks, M.M., Selvester, R.H., Swiryn, S.R.: Report of Committee on Nomenclature of Myocardial Wall Segments. in proc. Computerized Interpretation of Electrocardiograms VII proc. Engineering Foundation Conference (1984)

23. Startt/Selvester, R.H., Wagner, G.S., Ideker, R.E.: Myocardial Infaction. In: Macfarlane, P.W. and Lawrie, T.D.V., Editors.Comprehensive Electrocardiology: Theory and Practice in Health and Disease Pergamon Press pp. 565-629 (1989)

24. Gabriel, S., Lau, R.W., Gabriel, C.: The dielectric properties of biological tissues: II. Measurements in the frequency range $10 \mathrm{~Hz}$ to $20 \mathrm{GHz}$. Phys Med. Biol. 41(11), 2251-2269 (1996) 